\title{
PREVALÊNCIA DO USO DA ESCALA DE PERCEPÇÃO SUBJETIVA DE ESFORÇO BORG NOS EXERCÍCIOS FÍSICOS: REVISÃO INTEGRATIVA
}

\author{
PREVALENCE OF USING THE SUBJECTIVE BORG EFFORT \\ PERCEPTION SCALE IN PHYSICAL EXERCISES: INTEGRATIVE \\ REVIEW.
}

\author{
Maria Gabriely Queiroz ${ }^{1}$ \\ Danielle Franklin de Carvalho ${ }^{2}$ \\ Carla Campos Muniz Medeiros ${ }^{3}$ \\ Fernanda Caroline Tavares de Melo $^{4}$
}

\begin{abstract}
RESUMO: OBJETIVO: este estudo tem o intuito de conhecer acerca da prevalência do uso da escala de percepção de esforço subjetivo de Borg nos exercícios físicos. MÉTODOS: trata-se de uma revisão integrativa da literatura, realizada em quatro base de dados eletrônicas: Scientific Electronic Library Online (SciELO), National Library of Medicine (PubMed), Literatura Latino-Americana e do Caribe em Ciências da Saúde (LILACS), e Biblioteca Nacional em Saúde (BVS). Foram encontradas 216 artigos, com prevalência maior a base de dados BVS antes da aplicação dos critérios de inclusão e leitura avaliativa, após aplicação foram selecionados 3 para compor o estudo. RESULTADOS: A prevalência de estudos publicados foi na base de dados da SciELO sendo selecionados dois para compor o estudo, e um artigo da revista Brasileira de Prescrição e Fisiologia do Exercício, sendo perceptível a escassez de artigo com esta temática. A escala de Borg é um instrumento utilizado para quantificar através da percepção subjetiva de esforço aplicado no exercício, a intensidade do mesmo, ou seja, a força aplicada nos sistemas cardiopulmonar, musculares e fisiológicos. Estudos mostraram que a variação dos valores da percepção de esforço é um registro de tempo tolerável da atividade em diferentes temperaturas ambientais, sendo que, a elevação das taxas de Borg é auxiliada por
\end{abstract}

\footnotetext{
${ }^{1}$ Graduanda de enfermagem, Departamento de Enfermagem, UEPB, Campina Grande, PB, gaby$7741 @$ hotmail.com.

2 Doutora em Saúde da Criança e do Adolescente- UFPE, Mestrado em Saúde Pública e Pesquisadora do NEPE. Departamento de Enfermagem, UEPB, PB, daniellefranklin6@gmail.com.

${ }^{3}$ Doutora em Saúde da Criança e do Adolescente- FCM, Mestrado em Saúde da Criança e do Adolescente-FCM, Pesquisadora do NEPE. Departamento de Enfermagem, UEPB, PB, carlamunizmediros@hotmail.com.

${ }^{4}$ Graduada em Enfermagem-UEPB, Mestranda em Saúde Pública- UEPB, Campina Grande, PB, fernandactmelo@gmail.com.
} 
menor tempo de exaustão ao treino. Pesquisa efetuada no ano de 2015 objetivou o monitoramento da carga de treino através da escala de Borg, com 30 minutos de atividade moderada e 15 minutos de intensidade leve. Os participantes referiram maior cansaço e exaustão a partir do ciclo 3 ao 6 , no qual, a escala de Borg reconhece que, o treinamento foi eficiente para esta amostra que foi monitorada Os artigos referenciam a importância da escala de percepção subjetivo de esforço Borg, não só na prescrição de exercícios para evitar a fadiga e o cansaço ou para monitorar a intensidade aplicada naquele treino, mais também no auxilio a prescrição dos exercícios em pacientes que possuem patologias, evitando além da fadiga extrema, dores ou lesões por excesso de treino. CONCLUSÃO: O conhecimento obtido na literatura acerca da necessidade do uso da escala de Borg nos exercícios físicos a fim de melhorar o padrão de prescrição de treinos, oferecendo uma prescrição segura e eficaz durante os exercícios físicos são de suma importância.

Palavras chave: Escala, Esforço físico e Exercício.

ABSTRACT: OBJECTIVE: This study aims to know about the prevalence of the use of Borg's subjective effort perception scale in physical exercises. METHODOLOGY: This is an integrative literature review conducted in four electronic databases: Scientific Electronic Library Online (SciELO), National Library of Medicine (PubMed), Latin American and Caribbean Health Sciences Literature (LILACS), and National Health Library (VHL). A total of 216 articles were found, with higher prevalence in the VHL database before application of the inclusion criteria and evaluative reading, after application 3 were selected to compose the study. RESULTS: The prevalence of published studies was in the SciELO database and two were selected to compose the study, and an article from the Brazilian journal of Exercise Prescription and Physiology, noticing the scarcity of articles on this subject. The Borg scale is an instrument used to quantify, through subjective perception of exercise effort, the intensity of the exercise, ie, the force applied to the cardiopulmonary, muscular and physiological systems. Studies have shown that variation in exertion perception values is a tolerable time recording of activity at different ambient temperatures, and higher Borg rates are aided by shorter training exhaustion time. Research conducted in 2015 aimed to monitor the training load through the Borg scale, with 30 minutes of moderate activity and 15 minutes of light intensity. Participants reported greater tiredness and exhaustion from cycles 3 to 6 , in which the Borg scale recognizes that training was efficient for this monitored sample. The articles refer to the importance of the Borg subjective perception perception scale. Only in the prescription of exercises to prevent fatigue and tiredness or to monitor the intensity applied in that training, but also in helping the prescription of exercises in patients with pathologies, besides avoiding extreme fatigue, pain or injuries from over training. CONCLUSION: The knowledge obtained in the literature about the need to use the Borg scale in physical exercise in order to improve the training prescription pattern, offering a safe and effective prescription during physical exercise is of paramount importance.

Keywords: Scale, Physical Effort and Exercise. 


\section{INTRODUÇÃO}

O exercício físico é reconhecido como qualquer atividade física que promova o gasto calórico e aumento da aptidão física. Além de ser tratada como agente de proteção a saúde desde os anos de 1950, os benefícios que a mesma traz são inúmeros, que vão desde a melhora da condição física e qualidade de vida até a redução das incidências de doenças crônicas, melhorando perfil lipídico e a aumento da autoestima (POLISSENI., RIBEIRO, 2014).

Estudo realizado pela Pesquisa Nacional da Saúde (PNS) constatou que um a cada dois indivíduos não pratica a recomendação da Organização Mundial da Saúde que são 150 minutos de atividade com intensidade leve e moderada semanalmente, totalizando entre $20 \%$ a $30 \%$ de uma população considerada sedentária, que dispõe de grande probabilidade desenvolver uma doença crônica não transmissível (BRASIL, 2017).

A inatividade física chega ao século 21 devido a uma mudança de hábitos saudáveis (caminhada, pedalada, alimentação adequada) para hábitos não saudáveis, proveniente da, acessibilidade a tecnologia, o uso de computadores, smartphones, televisões, entre outras tecnologias; utilização de automóveis, no qual, não realiza mais a caminhada ou pedalada até o trabalho; excesso de trabalho, ansiedade; determinantes sociais, entre outros, culmina na prevalência da inatividade física (FREIRE et al., 2014; POLISSENI., RIBEIRO, 2014).

A falta de atividade física é vista como o quarto agente de risco para mortalidade, compondo um percentual de quase $30 \%$ de chance de morrer por qualquer causa. Pesquisas apontam que mais de $70 \%$ das pessoas morrem por Doenças Crônicas Não Transmissíveis (DCNTs) no Brasil, sendo a inatividade física um fator de risco comum dessas patologias (POLISSENI., RIBEIRO, 2014; RIBEIRO et al., 2016).

Tendo em vista que o excesso de peso por sua vez se relaciona com a falta de atividade física, contribuindo desta forma para o aumento da mortalidade devido 
as DCNTs. Pesquisa realizada com 280 pessoas constatou que mais de $28 \%$ da amostra era insuficientemente ativo com redução da prevalência de atividade física na faixa etária de 49 anos (POLISSENI., RIBEIRO, 2014; RIBEIRO et al., 2016).

O excesso de peso e a falta de exercícios físicos é uma epidemia mundial, no qual $13,9 \%$ da sociedade são obesas, sendo mais de $45 \%$ com excesso de peso e sedentarismo, informa a VIGITEL (Vigilância de Fatores de Risco e Proteção para Doenças Crônicas por Inquérito Telefônico). A obesidade pode ser classificada de acordo com o IMC Índice de Massa Corpórea, em que, é realizado um cálculo matemático (peso dividido por altura ao quadrado) o resultado irá definir se o estado nutricional é sobrepeso (IMC $\geq 25 \mathrm{~kg} / \mathrm{m}^{2}$ ) ou obesidade (IMC $\geq 30 \mathrm{~kg} / \mathrm{m}^{2}$ ). A pratica de atividades físicas associadas à escala de Borg permite que a intensidade do exercício seja mantida, garantindo que haja o gasto calórico suficiente para aquele treino (COSTA., VASCONCELOS., FONSECA, 2014; PINHEIRO., VIANA., PIRES, 2014).

Estudos mostram que mais de 450 milhões de pessoas no mundo possuem obesidade, relacionada há varias patologias como: diabetes, câncer, doenças cardiovasculares, acidente vascular cerebral, entre outras doenças crônicas. Inatividade física, alimentação não balanceada, estresse, ansiedade, tabagismo e alcoolismo são alguns fatores que contribuem para o excesso de peso (MALTA et al., 2014; ANDRADE et al., 2017).

Indivíduos que possuem certos tipos de doenças que causem certas limitações são necessários que haja prescrição adequada da carga e duração do treino, tendo em conta que a escala de Borg oferece o parâmetro ideal da intensidade necessária para aquela pessoa e treino adequado (MALTA et al., 2014; ANDRADE et al., 2017).

A escala de percepção subjetiva de esforço Borg, serve como instrumento para monitorar a intensidade do exercício realizado pelo indivíduo. É utilizado devido a fácil aplicabilidade, baixo custo e executável diariamente nas atividades. Esta escala permite mensurar o esforço percebido de maneira associada à frequência cardíaca, estabelecendo a intensidade do exercício praticado, pretendendo assegurar a prescrição correta e intensidade adequada do exercício evitando assim 
uma sobrecaga dos sistemas musculares e cardiopulmonares e complicações como dores, lesões e fadigas extremas (SANTOS et al., 2015).

A escala de Borg foi elaborada por Gunnar Borg nos anos de 1982, a princípio, englobando esta escala e outras escalas de percepção subjetiva de esforço, possuía a finalidade de quantificar a percepção de esforço obtida naquele exercício, atualmente, possui outras finalidades, como auxiliar na prescrição de exercícios e monitorização das cargas de treino (PINHEIRO., VIANA., PIRES, 2014).

São aplicadas em diversas faixas etárias, prevalentemente nos adultos, entretanto, na população pediátrica necessita de uma compreensão maior pela criança, em que, muitas vezes não entende ou consegue expressar o nível de esforço (MARTINS., ASSUMPÇÃO., SCHIVINSKI, 2014).

É uma ferramenta que segue uma ordem numérica crescente de 0 a 10, em que cada número relaciona-se com a sensação do esforço realizado no momento do exercício, vai desde muito, muito leve até o máximo, por exemplo, ajudando ao indivíduo a conhecer a intensidade e manté-la durante o exercício (MARTINS., ASSUMPÇÃO., SCHIVINSKI, 2014).

Salienta-se a necessidade de coletar evidências científicas objetivando conhecer acerca da prevalência do uso da escala de percepção de esforço subjetivo de Borg nos exercícios físicos, com vistas a contribuir para melhoria da prática de exercícios físicos, redução do peso e das doenças crônicas não transmissíveis.

\section{METODOLOGIA}

O presente trabalho consiste de uma revisão integrativa da literatura que permite a captação, avaliação crítica, e resumo do conhecimento sobre a temática abordada. Além de fornecer informações de maneira sistemática e organizada, através de pesquisas criteriosas e objetivas, promovendo a construção do conhecimento de modo resolutivo e eficaz de maneira a desenvolver outras pesquisas (ERCOLE., MELO., ALCOFORADO, 2014). 
Essa pesquisa foi produzida seguinte criteriosamente seis etapas: 1) escolha da questão norteadora da pesquisa (o uso da escala de BORG influencia nos exercícios físicos?); 2) estabelecimento dos critérios de inclusão e exclusão; 3) categorização dos estudos; 4) avaliação e análise dos estudos; 5) interpretação dos resultados e; 6) apresentação do conhecimento.

Foram incluídos no trabalho artigos que abordassem a temática proposta, nos idiomas inglês e português, publicados entre os anos de 2014 e 2019, disponíveis gratuitamente na íntegra e online. Foram excluídos artigos duplicados nas bases de dados.

O levantamento bibliográfico foi realizado nas bases de dados: Scientific Electronic Library Online (SciELO), National Library of Medicine (PubMed), Literatura Latino- Americana e do Caribe em Ciências da Saúde (LILACS), e Biblioteca Nacional em Saúde (BVS). Para nortear e facilitar a busca das publicações foram utilizados descritores cadastrados no DeCS (Descritores de Ciências da Saúde) que são: escala, esforço físico e exercício.

Quadro 01 - Distribuição dos artigos nas bases de dados antes e após a aplicação dos critérios de inclusão - Campina Grande, 2020.

\begin{tabular}{|c|c|c|c|}
\hline $\begin{array}{c}\text { Base de } \\
\text { dados }\end{array}$ & Estratégias de busca & $\begin{array}{c}\text { Publicações } \\
\text { encontradas }\end{array}$ & $\begin{array}{c}\text { Publicações } \\
\text { selecionadas }\end{array}$ \\
\hline Scielo & "Escala", "Esforço físico" e "Exercício" & 18 & 2 \\
\hline Pubmed & "Physical Exertion" and "escala" and "Exercise" & 2 & 0 \\
\hline Lilacs & "Physical Exertion" and "escala" and "Exercise" & 48 & 0 \\
\hline BVS & "Escala", "Esforço físico" e "Exercício" & 148 & 0 \\
\hline
\end{tabular}

A pesquisa foi realizada nas bases de dados, utilizado os descritores no idioma português na Scielo e BVS, e nas demais foi utilizado o idioma inglês com o booleano and. Foram encontradas 216 artigos, com prevalência maior na base de dados BVS antes da aplicação dos critérios de inclusão e leitura avaliativa.

Após aplicação dos critérios foram escolhidos três artigos para compor o estudo, sendo o terceiro retirado de uma revista não encontrada nas bases de dados, tendo em vista a relevância do conteúdo foi selecionada para construir a revisão. 


\section{RESULTADOS E DISCUSSÃO}

A prevalência de estudos publicados foi na base de dados da Scientific Electronic Library Online (SciELO) sendo selecionados dois para compor o estudo e um artigo da revista Brasileira de Prescrição e Fisiologia do Exercício, totalizando em três artigos na construção da revisão integrativa.

Tendo em vista que após a leitura criteriosa e analítica, não possuía nas outras bases de dados artigos que se encaixassem com a temática e os critérios de inclusão do estudo em questão, sendo perceptível a escassez de artigos com esta temática e a necessidade de mais estudos nesta área.

Quadro 02 - Disposição dos artigos selecionados de acordo com autores, ano, revista, título e objetivo respectivamente - Campina Grande, 2020.

\begin{tabular}{|c|c|c|c|c|}
\hline Autores & Ano & Revista & Título & Objetivo \\
\hline $\begin{array}{l}\text { ANDRADE, } \\
\text { et al. }\end{array}$ & 2017 & $\begin{array}{l}\text { Fisioterapia } \\
\text { Pesquisa }\end{array}$ & $\begin{array}{l}\text { A escala Borg CR-1c } \\
\text { adequada } \\
\text { quantificar } \\
\text { eintensidade } \\
\text { exercício aeróbico } \\
\text { mulheres } \\
\text { síndrome } \\
\text { fibromialgia. }\end{array}$ & 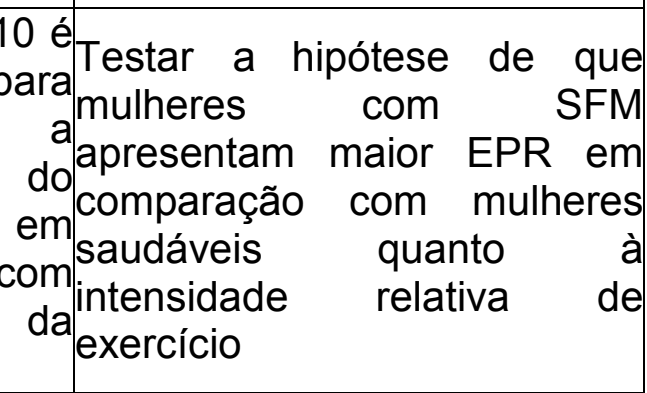 \\
\hline $\begin{array}{l}\text { PINHEIRO, } \\
\text { Fabiano; } \\
\text { VIANA, } \\
\text { Bruno; } \\
\text { PIRES, } \\
\text { Flavio. }\end{array}$ & 2014 & Motricidade & $\begin{array}{l}\text { Percepção subjetiva } \\
\text { esforço } \\
\text { marcadora da duraç } \\
\text { tolerável de exercício }\end{array}$ & 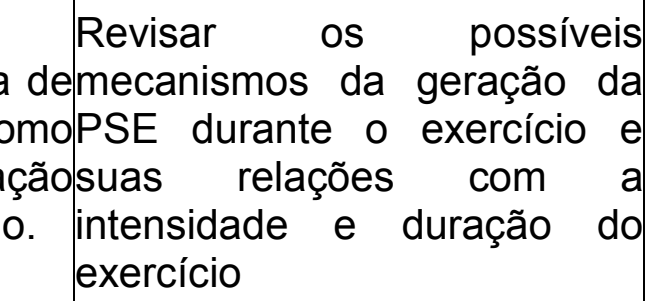 \\
\hline $\begin{array}{l}\text { SANTOS, } \\
\text { A. B. } \\
\text { et al. }\end{array}$ & 2015 & $\begin{array}{l}\text { Revista } \\
\text { Brasileira } \\
\text { Prescrição } \\
\text { Fisiologia } \\
\text { Exercício. }\end{array}$ & $\begin{aligned} & \begin{array}{l}\text { Monitoramento } \\
\text { carga interna }\end{array} \\
& \text { de } \text { trga treinamento através } \\
& \text { e } \text { escalas de percepç } \\
& \text { do } \text { subjetiva de bo } \\
& \text { 8oster e dor. }\end{aligned}$ & $\begin{array}{l}\text { daCentrou-se no monitoramento } \\
\text { deda carga de treino através de } \\
\text { deescalas de percepção } \\
\text { çãosubjetiva durante programa de } \\
\text { org, atividade física voltada a } \\
\text { saúde. }\end{array}$ \\
\hline
\end{tabular}


A escala de Borg é um instrumento utilizado para quantificar através da percepção subjetiva de esforço aplicado no exercício, a intensidade do mesmo, ou seja, a força aplicada nos sistemas cardiopulmonar, musculares e fisiológicos. Estudos mostraram que a variação dos valores da percepção de esforço é um registro de tempo tolerável da atividade em diferentes temperaturas ambientais, sendo que, a elevação das taxas de Borg é auxiliada por menor tempo de exaustão ao treino (PINHEIRO., VIANA., PIRES, 2014).

Outro estudo realizado em São Paulo com 24 pessoas que possuíam fibromialgia e 20 pessoas que não possuía, avaliou através da escala de Borg, a percepção de esforço realizado quando expostas a exercícios físicos. Resultou-se em que, as pessoas que tinham a síndrome perceberam a intensidade maior do exercício quando colocado à carga do treino, em vez daqueles que eram saudáveis. A partir do padrão obtido foi possível maior segurança nas prescrições dos exercícios em pacientes que possuem essa patologia (ANDRADE et al., 2017).

Pesquisa efetuada no ano de 2015 objetivou o monitoramento da carga de treino através da escala de Borg, submetendo 32 mulheres e 6 homens a seis ciclos de treino, com 30 minutos de atividade moderada e 15 minutos de intensidade leve. Os participantes referiram maior cansaço e exaustão a partir do ciclo 3 ao 6 , no qual, a escala de Borg reconhece que o treinamento foi eficiente para esta amostra que foi monitorada (SANTOS et al., 2015).

Os artigos referenciam a importância da escala de percepção subjetivo de esforço Borg, não só na prescrição de exercícios para evitar a fadiga e o cansaço ou para monitorar a intensidade aplicada naquele treino, mais também no auxilio a prescrição dos exercícios em pacientes que possuem limitações, patologias, evitando além da fadiga extrema, dores ou lesões por excesso de treino (SANTOS et al., 2015; ANDRADE et al., 2017). 


\section{CONCLUSÃO}

O conhecimento obtido na literatura acerca da necessidade do uso da escala de Borg nos exercícios físicos a fim de melhorar o padrão de prescrição de treinos, oferecendo uma prescrição segura e eficaz, evitando os problemas advindos de atividades de intensidade errada para determinada faixa etária, limitações entre outros, foi de suma importância.

É valido ressaltar que este estudo é de extrema necessidade para realização de outras pesquisas com base nesta temática, visto a escassez de estudo e pesquisas realizadas na esfera científica. 


\section{REFERÊNCIAS BIBLIOGRÁFICAS}

ANDRADE, C. P. et al. A escala Borg CR-10 é adequada para quantificar a intensidade do exercício aeróbico em mulheres com síndrome da fibromialgia. Fisioterapia e Pesquisa. São Paulo, vol. 24, n. 3, 2017.

BRASIL, Ministério da Saúde. Atividade Física. 2017.

COSTA, Miguel; VASCONCELOS, Ana; FONSECA, Maria. Prevalência de obesidade, excesso de peso e obesidade abdominal e associação com prática de atividade física em uma universidade federal. Revista Brasileira de Epidemiologia. São Paulo, vol. 17, n. 2, 2014.

ERCOLE, Flavia; MELO, Lais; ALCOFORADO, Carla. Revisão integrativa versus revisão sistemática. Revista Mineira de Enfermagem. Minas Gerais, vol. 18, n. 1, 2014.

FREIRE, R. S. et al. Prática regular de atividade física: estudo de base populacional no norte de minas gerais, Brasil. Revista Bras Med Esporte. Minas Gerais, vol. 20, n. 5, pág. 345- 349, 2014.

MALTA, D. C. Evolução anual da prevalência de excesso de peso e obesidade em adultos nas capitais dos 26 estados brasileiros e no Distrito Federal entre 2006 e 2012. Revista Brasileira de Epidemiologia. Minas Gerais, vol. 17, n. 1, pág. 267-276, 2014.

MARTINS, Renata; ASSUMPÇÃO, Maíra; SCHINVINSKI, Camila. Percepção de esforço e dispneia em pediatria: revisão das de avaliação. Medicina. Santa Catarina, vol. 47, n. 1, 2014.

PINHEIRO, Fabiano; VIANA, Bruno; PIRES, Flavio. Percepção subjetiva de esforço como marcadora da duração tolerável de exercício. Motricidade. São Paulo, vol. 10, n. 2, 2014.

POLISSENI, Maria. Exercício físico como fator de proteção para a saúde em servidores públicos. Revista Bras Med Esporte. Minas Gerais, vol. 20, n. 5, pág. 340-344, 2014.

RIBEIRO, A. Q. et al. Prevalência e fatores associados à inatividade física em idosos: um estudo de base populacional. Revista Bras Geriatra. Minas Gerais, vol. 19, n. 3, pág. 483- 494, 2016.

SANTOS, A. B. et al. Monitoramento da carga interna de treinamento através de escalas de percepção subjetiva de borg, foster e dor. Revista Brasileira de Prescrição e Fisiologia do Exercício. São Paulo, vol. 9, n. 52, pág. 121-128, 2015. 